\title{
PSEUDO-SEMISIMPLE RINGS
}

\author{
SAAD MOHAMED AND BRUNO J. MÜLLER ${ }^{1}$
}

\begin{abstract}
We characterize right-pseudo-semisimple rings (i.e. rings $R$ for which every right ideal is semisimple or isomorphic to $R$ ) which are split extensions of their right socle.
\end{abstract}

We call a ring $R$ (right) pseudo-semisimple if every right ideal not isomorphic to $R$ is semisimple. Principal right ideal domains and semisimple rings are trivial examples of pseudo-semisimple rings. Moreover, any local ring $R$ with Jacobson radical $J$ satisfying $J^{2}=0$ is a pseudo-semisimple ring. Mohamed and Singh [1] raised the following question: Does there exist a pseudo-semisimple ring with zero right singular ideal which is neither a principal right ideal domain nor semisimple? If such a ring does not exist, then by [1, Theorem 2.9$]$, a semiprime weak $q$-ring must be a principal right ideal ring or a right $q$-ring. In our (unsuccessful) attempt to find such an example we were led to consider rings in which the right socle splits off additively. For this class of rings we prove the following complete characterization of the pseudo-semisimple property.

THEOREM. Let $T$ be a subring and $S$ be an ideal of a ring $R$ such that $R=T \oplus S$ as additive groups. Then $R$ is right pseudo-semisimple with right socle $S$ if and only if $S^{2}=0, T$ is a principal right ideal domain, ${ }_{T} S$ is torsion free divisible and $S_{T}$ is semisimple.

It is clear that a ring of the type described by the above theorem can have zero right singular ideal only if it is a principal right ideal domain.

We start with the following.

LEMMA 1. Let $R$ be a ring with right socle $S$, and $T$ a subring of $R$ such that $R=S \oplus T$ as additive groups. If a proper right ideal $A$ is isomorphic to $R$, then $A+S \neq R$.

Proof. Assume that $A+S=R$. Since $A \simeq R, A=x R$ for some element $x \in R$ with right annihilator $x^{0}=0$. Therefore $1=x r+s, r \in R, s \in S$. Writing $r=t+s_{1}, t \in T, s_{1} \in S$ we get $1=x t+s_{2}$ where $s_{2}=x s_{1}+s \in S$. Also $x=t^{\prime}+s^{\prime}, t^{\prime} \in T, s^{\prime} \in S$. Then $1=t^{\prime} t+s_{3}$ where $s_{3}=s^{\prime} t+s_{2} \in S$. Hence $1-t^{\prime} t \in T \cap S=0$, and so $t^{\prime} t=1$. This proves that $t^{0}=0$, hence $(x t)^{0}=0$.

Let $K=s_{2}^{0} \cap S$. Then $S / K \simeq s_{2} S \subset s_{2} R$. Hence $S / K$ is a finite direct sum of simple $R$-modules. Since $(x t) k=k$ for all $k \in K$, and $(x t)^{0}=0$, we get

$$
S / K \simeq x t S / x t K=x t S / K \text {. }
$$

Thus $x t S=S$. Now $S=x t S \subset x S \subset x R=A$, which is a contradiction. This completes the proof.

Received by the editors September 15, 1980.

1980 Mathematics Subject Classification. Primary 16A48; Secondary 16A42, 16A52.

${ }^{1}$ This research was supported in part by the NSERC of Canada, Grant A 4033.

(c) 1982 American Mathematical Society 0002-9939/81/0000-0784/801.75 
COROLlary 2. Let $R$ be as in Lemma 1. Then any maximal right ideal isomorphic to $R$ is essential.

If $R$ is right pseudo-semisimple then every right ideal is either semisimple or principal. The following example shows that the converse is not true, in general.

EXAMPLE 3. A ring $R$ in which every right ideal is semisimple or principal, and no proper right ideal is isomorphic to $R$.

Let $V$ be an infinite-dimensional vector space over a field $F$, and let $S$ be the set of all finite rank linear transformations of $V_{F}$. Let $R=F \bigoplus S$. Then $S$ is the right socle of $R$. As $S$ is a maximal right ideal, every right ideal not contained in $S$ is a summand of $R$ and hence principal. Let $A$ be a proper right ideal. If $A \subset S$, then $A \not \not R$. On the other hand, $A \not \subset S$ implies that $A+S=R$ and $A \not \subset R$ by Lemma 1 .

The following is an example of a ring $R$ in which every nonsemisimple right ideal contains a copy of $R$, but $R$ is still not right pseudo-semisimple.

EXAMPLE 4. A ring $R$ in which every nonsemisimple right ideal contains a copy of $R$, but no maximal nonessential right ideal is isomorphic to $R$.

Let $V$ be a vector space with countable basis $\left\{b_{1}, b_{2}, \ldots\right\}$ over a field $F$, and let $S$ be the set of all finite rank linear transformation of $V_{F}$. Let $\Delta$ be the linear transformation defined by $\Delta b_{i}=b_{i+1}$. Let $F[\Delta]$ denote the ring of all polynomials in $\Delta$.

Let $f(\Delta)$ be a nonzero element in $F[\Delta]$. Then $f(\Delta)=\sum_{i=0}^{n} \alpha_{i} \Delta^{i}, \alpha_{i} \in F$, $\alpha_{n} \neq 0$. Let $\nu=\sum_{j=1}^{t} \eta_{j} b_{j} \in \operatorname{Ker} f(\Delta)$. Then

$$
\begin{aligned}
0 & =f(\Delta) \nu=\left(\sum_{i=0}^{n} \alpha_{i} \Delta^{i}\right)\left(\sum_{j=1}^{t} \eta_{j} b_{j}\right) \\
& =\sum_{l=1}^{n+t}\left(\sum_{h+k=l} \alpha_{h} \eta_{k}\right) b_{l} .
\end{aligned}
$$

This gives rise to the system of equations

$$
\begin{gathered}
\alpha_{n} \eta_{t}=0 \\
\alpha_{n} \eta_{t-1}+\alpha_{n-1} \eta_{t}=0 \\
\cdots \\
\alpha_{n} \eta_{1}+\alpha_{n-1} \eta_{2}+\cdots+\alpha_{0} \eta_{n+1}=0
\end{gathered}
$$

Thus $\eta_{i}=0, i=1,2, \ldots, t$. Hence $\operatorname{Ker} f(\Delta)=0$, and therefore $f(\Delta) V \simeq V$. This proves that $F[\Delta] \cap S=0$. Let $R=F[\Delta] \oplus S$. It is clear that $S=\operatorname{Soc} R_{R}$, and that $F[\Delta]$ is a subring of $R$. Let $\phi=g(\Delta)+s, s \in S, 0 \neq g(\Delta) \in F[\Delta]$. Since $s$ is of finite rank, Im $s \subset\left\langle b_{1}, b_{2}, \ldots, b_{m}\right\rangle$ for some integer $m$. Let $\psi=\Delta^{m}$. It is clear that $s \Delta^{m} V \subset\left\langle b_{1}, b_{2}, \ldots, b_{m}\right\rangle$ and $g(\Delta) \Delta^{m} V \subset\left\langle b_{m+1}, b_{m+2}, \ldots\right\rangle$. Now

$$
\begin{aligned}
\nu \in \operatorname{Ker} \phi \psi & \Rightarrow(g(\Delta)+s) \Delta^{m} \nu=0 \Rightarrow g(\Delta) \Delta^{m} \nu=-s \Delta^{m} \nu \\
& \Rightarrow g(\Delta) \Delta^{m} \nu \in\left\langle b_{1}, b_{2}, \ldots, b_{m}\right\rangle \cap\left\langle b_{m+1}, b_{m+2}, \ldots\right\rangle \\
& \Rightarrow g(\Delta) \Delta^{m} \nu=0 \\
& \Rightarrow \nu=0 \quad\left(\text { as } g(\Delta) \Delta^{m} \text { is a monomorphism }\right) .
\end{aligned}
$$

This implies that $(\phi \psi)^{0}=0$; indeed given $r \in R$

$$
\phi \psi r=0 \Rightarrow \phi \psi r V=0 \Rightarrow r V=0 \Rightarrow r=0 \text {. }
$$


Hence $\phi \psi R \simeq R$, and so $\phi R$ contains a copy of $R$. However, it follows by Corollary 2 that no maximal nonessential right ideal is isomorphic to $R$. (Note that maximal nonessential right ideals exist in abundance: If all maximal right ideals would be essential, then $S \subset J$, the Jacobson radical of $R$; therefore $S=S^{2} \subset S J=0$, a contradiction.)

Proof of The TheOREM. Assume that $R$ is right pseudo-semisimple with right socle $S$. Let $M$ be a maximal right ideal. If $M \not \subset R$, then $M$ is semisimple and hence $S=M$. On the other hand if $M \simeq R$, then $M$ is essential by Corollary 2, and so $S \subset M$. This shows that $S \subset \operatorname{Rad} R$ and hence $S^{2}=0$.

Let $K$ be a nonzero right ideal in $R / S$. Then $K=A / S$ for some right ideal $A$ properly containing $S$. Hence $A \simeq R$ and so $A$ is a principal right ideal. Therefore $K$ is a principal right ideal in $R / S$. This proves that $R / S$ is a principal right ideal ring.

Let $0 \neq t \in T$. Then $t R \simeq R$, and therefore $R=t^{0} \oplus B$ where $B \simeq t R \simeq R$. If $T^{0} \simeq R$, then $R \simeq R \oplus R$, which would imply that $R$ contains an infinite direct sum of copies of itself. Since such a right ideal cannot be isomorphic to $R$, it would be semisimple and hence $R$ would be semisimple, a contradiction. We conclude that $t^{0} \not \subset R$. Hence $t^{0} \subset S$, and therefore $t^{0} \cap T=0$. Therefore $R / S \simeq T$ is a domain.

Let $a \in R$ such that $a \notin S$. Then $a R=x R$ for some $x \in R$ with $x^{0}=0$. As $x R+S \supsetneqq S, x R+S=y R$ for some $y \in R$ with $y^{0}=0$. Then $y=x r+s$, $r \in R$ and $s \in S$. Let $r=t+s_{1}$. Then $y=x t+s_{2}, s_{2} \in S$. Now $x \in y R$ implies $x=y r^{\prime}, r^{\prime} \in R$. Let $r^{\prime}=t^{\prime}+s^{\prime}, t^{\prime} \in T$ and $s^{\prime} \in S$. Then $y=y t^{\prime} t+s_{3}, s_{3} \in S$. Hence $y\left(1-t^{\prime} t\right) \in S$. As $R / S$ is a domain and $y \notin S, 1-t^{\prime} t \in S$ and therefore $1-t^{\prime} t \in S \cap T=0$. Hence $t^{0}=0$ and then $(x t)^{0}=0$.

Since $S \subset y R, S=y B$ for some right ideal $B$ of $R$. However $y b \in S$ and $y \notin S$ implies $b \notin S$ as $R / S$ is a domain. Hence $B=S$ and we get $S=y S$. Let $K=s_{2}^{0} \cap S$ and consider the diagram

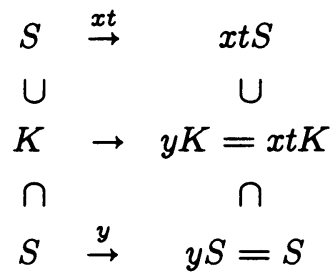

Then

$$
x t S / y K=x t S / x t K \simeq S / K \simeq y S / y K=S / y K .
$$

Since $S / K$ is a finite sum of simple modules, $x t S=S$. Then

$$
S \supset a S=a R S=x R S \supset x t S=S .
$$

Hence $a S=S$. In particular $t S=S$ for every $t \in T$. Therefore ${ }_{T} S$ is divisible.

Let $u$ be a nonzero element in $T$. Let $u f=0$ for some $f \in S$. Since $u R \simeq R$, there exist $\nu \in T$ and $g \in S$ such that $(u(\nu+g))^{0}=0$. The divisibility of ${ }_{T} S$ implies that $f=\nu h$ for some $h \in S$. As $S^{2}=0$, we have $g h=0$. Then

$$
u(\nu+g) h=u \nu h+u g h=u f=0 .
$$

Therefore $h=0$, and hence $f=0$. Therefore ${ }_{T} S$ is torsion free. 
Let $N$ be a $T$-submodule of $S$. Since $S^{2}=0, N$ is an $R$-submodule of $S$. Then $N$ is a summand of $S$ as an $R$-module and of course as a $T$-module. Therefore $S_{T}$ is semisimple.

Conversely, assume that $R=T \bigoplus S$ satisfies the conditions. It is clear that $S$ is the right socle of $R$. Let $A$ be a right ideal of $R$ which is not semisimple. Then $A$ contains an element $t+s$ where $t \neq 0$. Since $S^{2}=0$ and ${ }_{T} S$ is divisible, we get

$$
A \supset(t+s) S=t S=S \text {. }
$$

As $R / S$ is a principal right ideal domain,

$$
A=t_{1} R+S=t_{1} R+t_{1} S=t_{1} R \text {. }
$$

Let $t_{2}+s_{2} \in R$ be such that $t_{1}\left(t_{2}+s_{2}\right)=0$. Then

$$
t_{1} t_{2}=-t_{1} s_{2} \in T \cap S=0 .
$$

Since $T$ is a domain and ${ }_{T} S$ is torsion free, $t_{2}=s_{2}=0$. Hence $t_{1}^{0}=0$, and therefore $A=t_{1} R \simeq R$.

We conclude this paper by giving an example of a right pseudo-semisimple ring which is not left pseudo-semisimple.

EXAMPLE 5. Let $K$ be a field, $K[x]$ the ring of all polynomials over $K$ and $K(x)$ the field of rational functions. Let $T=K[x]$ and $S=K(x)$. For $t \in T$ and $s \in S$ define $t s$ as ordinary multiplication in $S$, and $s t$ as multiplying $s$ by the constant term in the polynomial $t$. It is clear that ${ }_{T} S$ is torsion free divisible and $S_{T}$ is semisimple. Let $R=\{(t, s): t \in T, s \in S\}$. Define addition and multiplication in $R$ by

$$
\begin{aligned}
& \left(t_{1}, s_{1}\right)+\left(t_{2}, s_{2}\right)=\left(t_{1}+t_{2}, s_{1}+s_{2}\right), \\
& \left(t_{1}, s_{1}\right) \cdot\left(t_{2}, s_{2}\right)=\left(t_{1} t_{2}, t_{1} s_{2}+s_{1} t_{2}\right) .
\end{aligned}
$$

With respect to these operations, $R$ is a ring. Identifying $T$ and $S$ with their respective images in $R$, we find that $T$ is a subring of $R, S^{2}=0$ and $R / S \simeq T$ a principal right ideal domain. It follows by the above theorem that $R$ is right pseudo-semisimple with right socle $S$.

Since $\operatorname{Soc}\left({ }_{T} S\right)=0$ and $\operatorname{Soc}\left({ }_{T} T\right)=0$, we get $\operatorname{Soc}\left({ }_{R} R\right)=0$. Therefore if $R$ is left pseudo-semisimple, then every left ideal of $R$ is isomorphic to ${ }_{R} R$, which would imply that $R$ is a domain, a contradiction.

\section{REFERENCES}

1. Saad Mohamed and Surjeet Singh, Weak q-rings with zero singular ideal, Proc. Amer. Math. Soc. 76 (1979), 25-30.

\section{DEPARTMENT OF MATHEMATICS, KUWAIT UNIVERSITY, KUWAIT}

DEPARTMENT OF MATHEMATICS, MCMASTER UNIVERSITY, HAMILTON, ONTARIO L8S 4K1, CANADA 